\title{
On the Effect of Rapid Area Change in Perching-Like Maneuvers
}

\author{
Delyle T. Polet, ${ }^{*}$ Tyler Christensen ${ }^{\dagger}$ and David E. Rival ${ }^{\ddagger}$ \\ University of Calgary, Calgary, AB, T2N 1N4, Canada \\ Gabriel D. Weymouth ${ }^{\S}$ \\ University of Southampton, Southampton, S017 1BJ, UK
}

\begin{abstract}
A perching bird is able to rapidly decelerate at a high angle of attack while maintaining lift and control. However, the underlying aerodynamic mechanism is poorly understood. We perform a study on a simultaneously decelerating and pitching airfoil section as a simple perching model. First, we use analytic arguments to establish the inertial non-circulatory force on a wing section, and its dependence on the shape change number, $\bar{\tau}^{*}$, the ratio between the rate of change of frontal dimension and the initial translational velocity. Next, we report that forces measured on a towed and pitched airfoil at $R e=22000$, and forces from simulations at $R e=2000$, are found to be well above non-circulatory predictions, exhibiting high lift and drag. Flow-field visualizations, both from Particle Image Velocimetry and simulations, reveal strong coherent vortical structures in the wake and near the body. At a higher shape change number, vortices in the wake convect more quickly than at a lower shape change number, generating higher drag. Additionally, separation of the LEV and positive vorticity near the body is reduced at a higher shape change number, increasing lift. Thus wake manipulation through rapid area change provides a means through which a perching bird can maintain high lift and drag simultaneously while slowing to a controlled stop.
\end{abstract}

\section{Nomenclature}

a Acceleration, $\mathrm{m} / \mathrm{s}^{2}$

BDIM Boundary Data Immersion Method

c Airfoil chord, m

$C_{d} \quad$ Drag coefficient

$C_{l} \quad$ Lift coefficient

$C_{F} \quad$ Chord-normal force coefficient

$F \quad$ Force per unit span, N/m

LEV Leading-edge Vortex

$m_{a} \quad$ Added mass

$p \quad$ Pressure, $\mathrm{Pa}$

PIV Particle Image Velocimetry

$q \quad$ Fluid speed, $\mathrm{m} / \mathrm{s}$

$r \quad$ Distance between vortices, $m$

$\mathbf{r}$ Position of the frame of reference

Re Reynolds number
$S \quad$ Submerged span, $\mathrm{m}$

SV Starting Vortex

$t^{*} \quad$ Non-dimensional time

$T \quad$ Period of deceleration and pitching, $\mathrm{s}$

$U$ Instantaneous velocity, $\mathrm{m} / \mathrm{s}$

$U_{0} \quad$ Initial velocity, $\mathrm{m} / \mathrm{s}$

$V \quad$ Shape change velocity, $\mathrm{m} / \mathrm{s}$

$\mathbf{V}_{\mathbf{0}} \quad$ Velocity of the frame of reference

$x_{p}^{*} \quad$ Non-dimensional pivot position from leading edge

$\alpha \quad$ Angle of attack

$\Gamma \quad$ Vortex circulation, $\mathrm{m}^{2} / \mathrm{s}$

$\rho \quad$ Fluid density, $\mathrm{kg} / \mathrm{m}^{3}$

$\sigma^{*} \quad$ Deflation scaling parameter

$\bar{\tau}^{*} \quad$ Time-averaged shape change number

$\boldsymbol{\Omega} \quad$ Angular velocity of the frame of reference

\footnotetext{
*Graduate Student, Mechanical Engineering, d.polet@ucalgary.ca, AIAA Student Member.

${ }^{\dagger}$ Undergraduate Student, Mechanical Engineering

¥Associate Professor, Mechanical Engineering, derival@ucalgary.ca, AIAA Member.

$\S$ Lecturer, Southampton Marine and Maritime Institute, G.D.Weymouth@soton.ac.uk.
} 


\section{Introduction}

Birds achieve quick, precise maneuvers, such as banking, braking, and perching, by rapidly changing wing area while pitching and accelerating their wings. Flapping-type Micro Aerial Vehicles (MAVs), often inspired by birds, have been developed, but it is difficult for these machines to perform similar rapid maneuvers. Rapid area change of control surfaces is an avenue yet to be explored in these vehicles, and its careful application could narrow the performance gap between small man-made and natural flyers.

To perch successfully, birds must rapidly decelerate, requiring large drag forces. However, the loss of speed and high angle of attack may lead to a loss of lift and control. ${ }^{1}$ Yet birds are quite adept at perching, and the entire maneuver is well-controlled. ${ }^{2,3}$ The aerodynamic means by which birds are able to maintain lift and drag simultaneously as the animal slows is as yet unknown.

During perching, birds rapidly rotate and expand their wings, quickly increasing frontal area. ${ }^{2,3}$ Recent studies $^{4-6}$ have drawn attention to the phenomenon of rapid area change of bodies in motion in fluid mediums, showing that rapid area change can have a pronounced effect on drag, vorticity and added mass. For instance, Wibawa et al. ${ }^{4}$ showed that boundary-layer vorticity is rapidly shed into the fluid for a vanishing airfoil. Weymouth and Triantafyllou ${ }^{5}$ found that a shrinking cylinder recovers added-mass energy and exhibits significant thrust during its collapsing phase. Weymouth and Triantafyllou ${ }^{6}$ showed that a squid-like deflating body achieves much higher rates of acceleration and reduced drag as compared to non-deflating bodies.

These studies have all looked at the fluid dynamics of a body undergoing a rapid decrease in area. However, the case of a body experiencing a rapid increase in area has seen comparatively little attention, despite the potential to inform biological and technological designs.

Though bird perching is a three-dimensional problem, we can gain insight into the fluid dynamics of rapid wing area change by considering an abstracted, two-dimensional configuration. Through analytical, numerical and experimental methods, we show that the rapid change of an airfoil area is able to promote high lift while also exhibiting high drag: precisely the combination required for controlled perching.

\section{Analytical Arguments}

Consider an airfoil initially at an angle of attack $\alpha=0^{\circ}$ and moving at a constant forward velocity $U_{0}$. As a simple model of a bird's wing during perching maneuvers, this airfoil section is made to rapidly rotate perpendicular to the translation direction, that is $\alpha=90^{\circ}$, while simultaneously decelerating at a rate $a=U_{0} / T$ to a full stop over time period $T$. This system and its kinematics are sketched in figure 1 .

We parametrize the magnitude of the geometry change as $\bar{\tau}^{*}=V / U_{0}$, where $V \equiv c / T$ is the average speed at which the frontal width of the airfoil increases over the course of the maneuver. Substitution shows that

$$
\bar{\tau}^{*}=\frac{V^{2}}{a c},
$$

matching the shape change number defined in Weymouth and Triantafyllou, ${ }^{6}$ though we avoid their choice of $\Xi$ for notation. Note that $\bar{\tau}^{*}$ is the time-average of the instantaneous relative velocity of shape change, $\tau^{*} \equiv \frac{V(t)}{2 U(t)}$, over the maneuver.

Previous studies have shown that added-mass forces are important or even dominant in some rapid area change problems at large $\bar{\tau}^{*}{ }^{5,6}$ Potential flow theory provides a means to model the added-mass force on airfoils. If a velocity potential $\Phi$ for flow past an airfoil is found, then the pressure difference between two points can be computed using the unsteady Bernoulli equation,

$$
\frac{\Delta p}{\rho}=\frac{1}{2}\left(q_{2}^{2}-q_{1}^{2}\right)-\left(\mathbf{V}_{\mathbf{0}}+\boldsymbol{\Omega} \times \mathbf{r}\right) \cdot\left(\nabla \phi_{2}-\nabla \phi_{1}\right)+\frac{\partial}{\partial t}\left(\phi_{2}-\phi_{1}\right) .
$$

In Eq. (2), subscripts 1 and 2 refer to points along the streamline of interest. To calculate the force on the airfoil, we need only determine the potential at the airfoil surface and integrate using Eq. (2). The potential owing to translation and reorientation of the airfoil can be superimposed with the potential owing to the rapid rotation of the airfoil, yielding the final potential.

To calculate the added-mass force, we require only the velocity potential due to the instantaneous impermeable boundary condition at the body surface. ${ }^{7}$ Thus, we avoid considerations of bound circulation, flow separation or circulation in the wake. We will show that $\bar{\tau}^{*}$ parameterizes the added-mass force, but that this effect alone cannot generate the forces required for controlled perching. 
a)

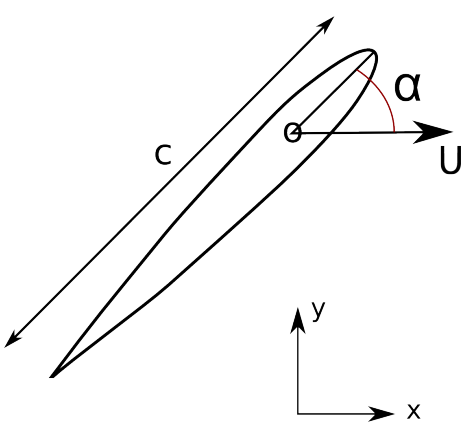

b)

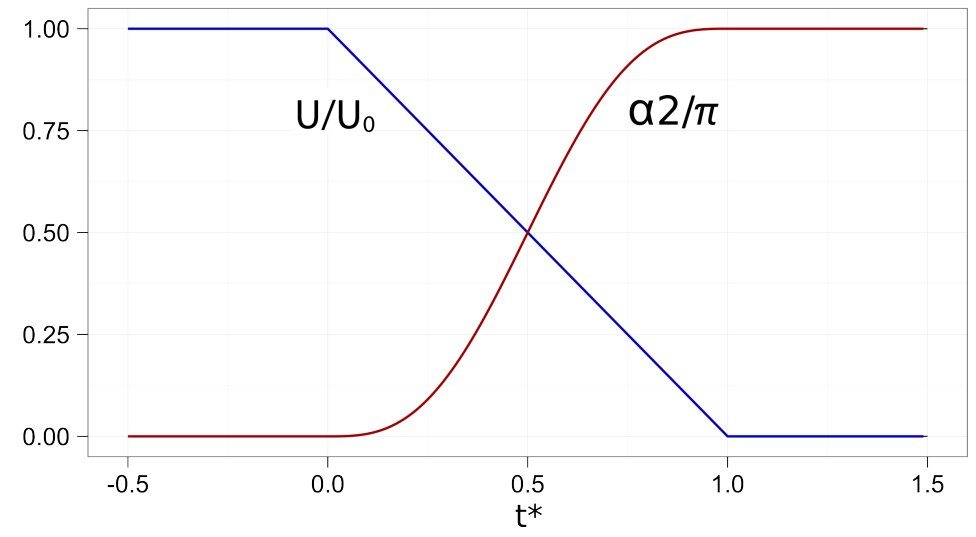

Figure 1: (a) A diagram showing the NACA0012 airfoil section with chord $c$ and pivot point $o$ at the onesixth chord position. The instantaneous velocity $U$ at the pivot and angle of attack $\alpha$ are prescribed. (b) The kinematics of the model problem plotted as a function of time $t^{*}=t / T$, where $T$ is the time scale of the maneuver. The velocity is scaled by the initial value $U_{0}$, while the angle is scaled by the final value $\pi / 2$. $\alpha$ is varied with a cycloidal function given in Eq. (14).

\section{A. Potential flow solutions and the non-circulatory force}

We first consider a flat-plate airfoil of chord $c$ slowly rotating in a uniform ambient of flow speed $U$, such that $c \dot{\alpha} \ll U$. The potential at the surface of the airfoil $(y=0)$ is

$$
\Phi_{a}(x, 0)=\frac{U c}{2}\left(\frac{2 x}{c} \cos \alpha \pm \sin \alpha \sqrt{1-(2 x / c)^{2}}\right),
$$

where $x$ is the position along the chord ( $x=0$ is the location of the half chord). The positive square root refers to the top surface and the negative square root to the bottom surface. In the unsteady case, $\alpha$ and $U$ are time-dependent. However, Eq. (3) satisfies the boundary conditions at each instant (ignoring the rotational speed) even when these parameters are time-dependent. Thus, Eq. (3) is the unsteady surface potential for a decelerating and slowly-rotating, flat-plate airfoil.

For a flat-plate airfoil quickly rotating about a point at a distance $c x_{p}^{*}$ from its leading edge in an otherwise quiescent fluid, the potential at the airfoil surface is

$$
\Phi_{b}(x, 0)= \pm \dot{\alpha} \frac{c^{2}}{4}\left[1-2 x_{p}^{*}+\frac{x}{c}\left(2\left(1-2 x_{p}^{*}\right)^{2}-1\right)\right] \sqrt{1-(2 x / c)^{2}},
$$

where, again, the positive portion refers to the top surface and the negative portion to the bottom surface. Because of the linearity of potential flow solutions, Eqs. (3) and (4) may be superimposed to yield the total potential in the absence of circulation. We may now use Eq. (2) to calculate the pressure gradient between the top and bottom surfaces, which in turn generates the force acting normal to the plate. The result is

$$
F_{\perp}=\rho \frac{\pi c^{2}}{4}\left[\dot{\alpha} \cos (\alpha) U+\sin (\alpha) \dot{U}+c \ddot{\alpha}\left(1 / 2-x_{p}^{*}\right)\right],
$$

with the positive direction defined as towards the suction side. $\rho \pi c^{2} / 4$ has units of mass per length, and is the maximum added mass per unit span. Thus, the non-circulatory force in Eq. (5) is the total added-mass force for this potential model, but we will use the term "non-circulatory force" henceforth to refer specifically to Eq. (5) and its normalization. The total normalized non-circulatory force is

$$
C_{F}=\frac{\pi c}{2 U_{\infty}^{2}}\left[\dot{\alpha} \cos (\alpha) U+\sin (\alpha) \dot{U}+c \ddot{\alpha}\left(1 / 2-x_{p}^{*}\right)\right] .
$$

Note that $C_{F}$ maps to lift and drag as

$$
C_{L}=C_{F} \cos \alpha, \quad C_{D}=C_{F} \sin \alpha .
$$


For small $\alpha$ and steady flow $(\dot{U}=0)$, Eq. (6) generates a lift,

$$
C_{L}=\frac{\pi c}{2 U_{\infty}^{2}}\left(\dot{\alpha} U-\frac{c\left(2 x_{p}^{*}-1\right) \ddot{\alpha}}{2}\right),
$$

which is identical to the added-mass lift originally derived by Theodorsen ${ }^{8}$ for small angles of attack in the absence of plunge, as recently presented in $\mathrm{Ol}$ et al. ${ }^{9}$ Therefore, Eq. (6) can be thought of as the extension of the non-circulatory portion of Theodorsen's force model to large angles of attack and unsteady translation, without plunge.

\section{B. Dependence of non-circulatory forces on shape change number}

Next we establish how Eq. (6) depends on $\bar{\tau}^{*}$. Over the course of a rotation from $\alpha=0^{\circ}$ to $90^{\circ}$ in time $T$, the magnitude of $\dot{\alpha}$ can be estimated as

$$
\dot{\alpha} \sim \frac{\pi}{2 T}=\bar{\tau}^{*} \frac{\pi U_{0}}{2 c} .
$$

Similarly, $\ddot{\alpha}$ can be estimated as

$$
\ddot{\alpha} \propto \frac{\pi}{2 T^{2}}=\bar{\tau}^{* 2} \frac{\pi U_{0}^{2}}{2 c^{2}},
$$

while we can write $\dot{U}$ as

$$
\dot{U}=\bar{\tau}^{*} \frac{U_{0}^{2}}{c},
$$

showing that $\bar{\tau}^{*}$ also parametrizes the rate of rotation, rotational acceleration and translational acceleration. From these relations, we rewrite Eq. (6) as

$$
C_{F}=f(t ; \alpha) \bar{\tau}^{*}+g(t ; \alpha) \bar{\tau}^{*}+h\left(t, x_{p}^{*}\right) \bar{\tau}^{* 2},
$$

where $f, g$ and $h$ are functions of time, based on the kinematics of the problem, and refer to forces from the entrainment, linear acceleration and rotational acceleration of added mass, respectively. $h$ is also a function of the pivot position $x_{p}^{*}$, and will be maximal at $x_{p}^{*}= \pm 1$ and minimal (zero) at $x_{p}^{*}=1 / 2$. Eq. (7) in combination with Eq. (12) shows that the lift and drag should scale by no more than $\bar{\tau}^{* 2}$, in the absence of circulatory forces.

\section{Comparison of added mass in expanding versus shrinking rapid area change problems}

Though the non-circulatory forces in Eq. (5) depend on $\dot{\alpha}$, we can gain insight by first considering the translational part. The added-mass reaction force inline with the body motion can be written as

$$
F_{A M}=-\frac{\partial}{\partial t}\left(m_{a} u\right)=-\dot{m}_{a} u-m_{a} \dot{u}
$$

where $u$ is the speed of the body and is positive in the direction of travel. By comparing Eq. (13) to (5), we notice that the first term refers to the entrainment of added-mass momentum $\left(\dot{m}_{a} u\right)$ and the second to the acceleration of the added mass $\left(m_{a} \dot{u}\right)$. Weymouth and Triantafyllou ${ }^{6}$ found that an accelerating and deflating prolate spheroid saw a reduction in added mass through its maneuver $\left(\dot{m}_{a}<0\right)$. This had a twofold effect in view of Eq. (13). The first was in achieving additional thrust by using the shed added mass as a propellant (the $-\dot{m}_{a} u$ term). The second was in reducing parasitic drag throughout the maneuver (the $-m_{a} \dot{u}$ term). Both effects were beneficial for the performance goal of high acceleration.

For a bird trying to achieve rapid deceleration, however, there is a tradeoff. On the one hand, entrainment of added-mass from frontal area expansion $\left(\dot{m}_{a}>0\right)$ would create drag through $-\dot{m}_{a} u$. But this same entrainment would increase the total added mass in time, generating a strong parasitic thrust late in the maneuver via $-m_{a} \dot{u}$. The rotational acceleration term in Eq. (5) cannot fully compensate in drag, as $\ddot{\alpha}$ must be positive early in the maneuver at low angles of attack (generating lift) and negative late in the maneuver at high angles of attack (generating thrust). Therefore, according to this perching model, a bird needs to generate forces in other ways to achieve high drag.

In this analytical treatment, we have ignored circulatory effects, such as separation and wake effects. These are more difficult to treat analytically, and therefore we will now explore them empirically instead. 
We performed a simple perching maneuver with an airfoil in the laboratory at different $\bar{\tau}^{*}$. Forces on the airfoil were measured directly, while the flowfield was measured using Particle Image Velocimetry (PIV) to evaluate circulatory effects. Two-dimensional simulations at a lower Reynolds number were also performed to assess Reynolds number effects, as well as to achieve near-body information unavailable with PIV.

\section{Experimental Setup}

All laboratory experiments were performed in a free-surface towing facility at the University of Calgary. The water channel test section is $38.6 \mathrm{~cm}$ wide, and water level was maintained at a $42 \mathrm{~cm}$ depth. An aluminum NACA0012 airfoil with $48 \mathrm{~mm}$ chord was positioned midway between the channel walls in a vertical orientation (figure $2 \mathrm{~b}$ and $2 \mathrm{c}$ ) and pierced the free surface from above. The airfoil tip was $4 \mathrm{~mm}$ from the bottom of the test section to reduce tip effects. Thus the airfoil had a submerged span of $416 \mathrm{~mm}$ and an aspect ratio of 8.7. The high aspect ratio further justifies the assumption of two-dimensional flow. A plastic skim plate of $112 \mathrm{~mm}$ diameter and $2 \mathrm{~mm}$ thickness was secured to the airfoil and sat $3 \mathrm{~mm}$ below the water surface. This skim plate eliminated the formation of free-surface funnel vortices. The airfoil was attached at one end to an ATI Gamma force/torque balance. The force balance was attached to a two-phase stepper motor with $0.9^{\circ}$ step angle. The pitching axis was $c / 6$ from the leading edge. The apparatus was fixed to a Parker linear traverse, which ran along the length of the water channel.

The airfoil first established a steady-state condition at $\alpha=0^{\circ}$ and speed $U_{0}=0.45 \mathrm{~m} / \mathrm{s}$ for at least 10 chord lengths before beginning rotation and deceleration. The Reynolds number at the beginning of the maneuver is therefore $R e=22000$, in the range relevant for small, highly-maneuverable birds. The start of unsteady kinematics was synced through an induction sensor at a fixed position on the traverse track, which detected the passing traverse stage. The kinematics follow those shown in figure 1. Rotation of the airfoil was set at a prescribed rate according to the cycloidal function,

$$
\alpha(t)=\frac{\pi}{2}\left[t^{*}-\frac{\sin \left(2 \pi t^{*}\right)}{2 \pi}\right], 0 \leq t \leq 1,
$$

where $t^{*}=t / T=t V / c$ is the non-dimensional time. This function was so chosen because it has finite jerk throughout. The translational deceleration was constant and was determined by the shape change number (Eq. 11).

From Eq. (6), the prescribed kinematics were expected to yield a non-circulatory force perpendicular to the plate,

$$
C_{F}=\frac{\pi^{2}}{2}\left[\bar{\tau}^{*}\left(1-t^{*}\right) \sin ^{2}\left(\pi t^{*}\right) \cos \alpha-\bar{\tau}^{*} \frac{\sin \alpha}{\pi}+\bar{\tau}^{* 2} \frac{\pi}{3} \sin \left(2 \pi t^{*}\right)\right] .
$$

Using the relations in (7), the resulting lift and drag will be compared with measured forces in figure 3 .

\section{A. Force Measurements}

Force measurements were taken at a $1000 \mathrm{~Hz}$ sample rate (16bit sample depth). Recorded force data was averaged across 10 trials for each test case. The force measurements were repeated for the same kinematics once water had been drained from the channel in order to measure the non-hydrodynamic inertia of the system. Force data was transformed from an airfoil-fixed frame to a lab-fixed frame, and the inertia was subtracted. The transformed data was further smoothed with a two-degree Butterworth low-pass filter. Filter frequency cutoff was chosen as $4 \mathrm{~Hz}$ for $\bar{\tau}^{*}=1 / 32$ and $10 \mathrm{~Hz}$ for $\bar{\tau}^{*}=1 / 2$. Filter frequency cutoffs for intermediate $\bar{\tau}^{*}$ were determined by interpolating linearly between these values. This method was found to best preserve peaks while eliminating noise. Force measurements were synchronized through the same induction sensor on the traverse track that triggered the deceleration.

Force measurements were also performed on the same airfoil at a constant speed $(0.45 \mathrm{~m} / \mathrm{s})$ with the angle of attack varying between trials. $\alpha$ was varied from $0^{\circ}$ to $5^{\circ}$ with $1^{\circ}$ increments, and from $5^{\circ}$ to $90^{\circ}$ with $5^{\circ}$ increments using a rotational stage (figure 2c). The same measurements were performed for negative values of $\alpha$ to assess the symmetry of the setup. These measurements were used to make quasi-steady predictions for force measurements. Force coefficients were computed as

$$
C_{F}=\frac{2 F}{\rho c S} \frac{U(\alpha)^{2}}{U_{0}^{4}},
$$



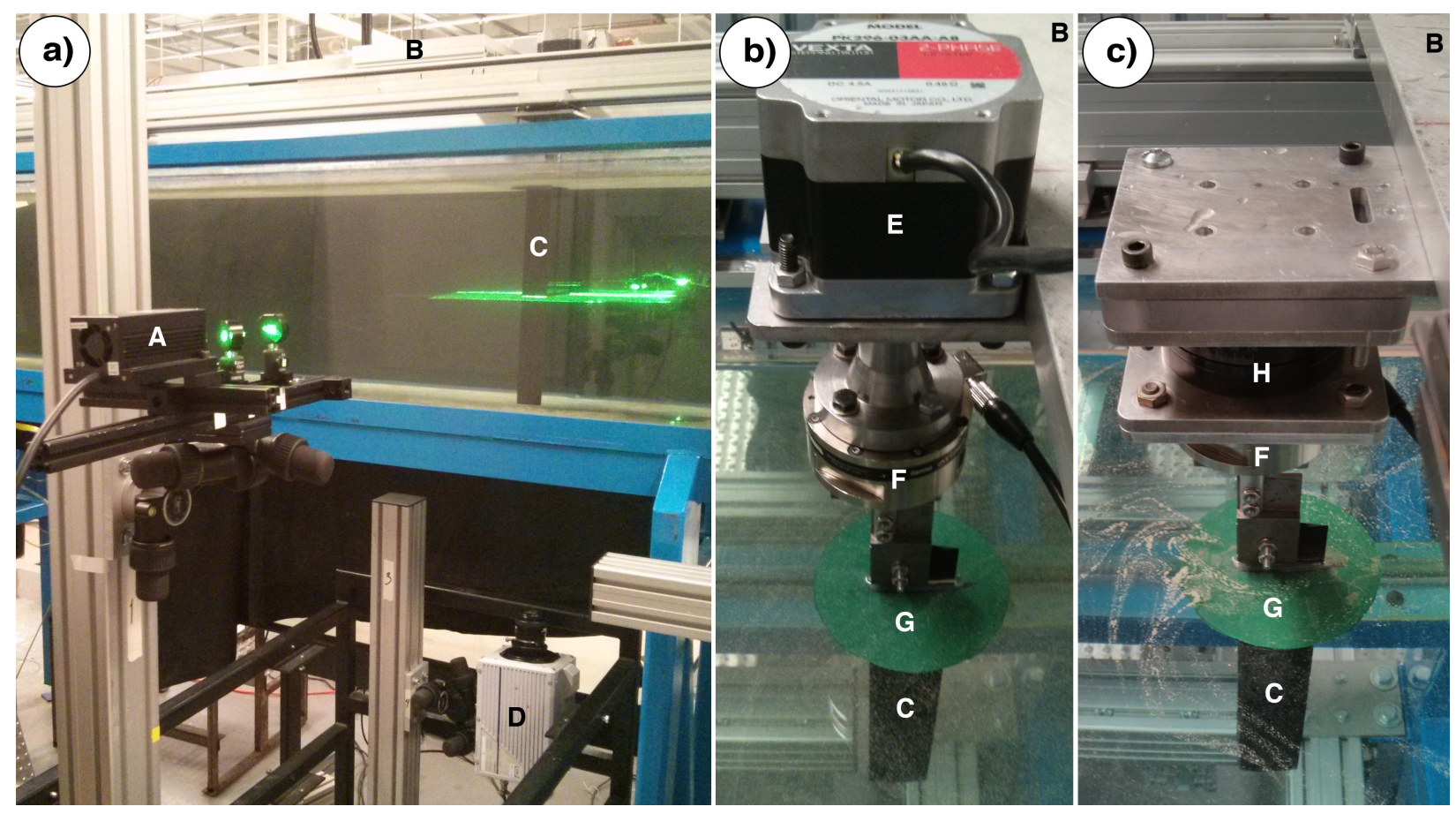

Figure 2: (a) PIV setup, showing a laser (A) projecting a laser sheet into the water tunnel from the side. The airfoil (C), which is attached to the traverse stage (B), is translated through the laser sheet with prescribed kinematics. A Photron SA4 high-speed camera (D) filmed the maneuver through the glass bottom of the water channel. (b) The airfoil attachment used for PIV and force measurements. The airfoil (C) was attached to a 6-component force/torque balance $(\mathrm{F})$, which in turn was attached to a stepper motor $(\mathrm{E})$. A plastic skim plate $(\mathrm{G})$ prevented the formation of free surface vortex funnels. The entire attachment was secured to the stage of a linear traverse (B), which sat on top of the water channel. (c) The airfoil attachment used for steady force measurements. The same airfoil (C), skim plate $(\mathrm{G})$, and force balance (F) attachment as the unsteady setup (b) was used. The force balance was attached to a rotational stage $(\mathrm{H})$, which in turn was secured to the traverse stage (B).

where $F$ is the measured force, $U(\alpha)$ is the speed of the airfoil at a particular angle of attack in the unsteady case, and $U_{0}$ is the starting speed.

\section{B. Particle Image Velocimetry (PIV)}

A $1 \mathrm{~W}$, continuous-wave laser $(\lambda=532 \mathrm{~nm})$ projected a laser sheet into a plane orthogonal to the airfoil (figure 2a). This laser sheet was $21.1 \mathrm{~cm}$ above the water channel floor. The water was seeded with silver-coated, hollow glass spheres of $100 \mu \mathrm{m}$ diameter. These particles have a Stokes number of approximately $2.4 \times 10^{-3}$, and therefore were assumed to accurately follow the fluid flow. As the airfoil passed through the laser sheet, a Photron SA4 high-speed camera $(1024 \times 1024$ pixel resolution) captured images at 250 frames per second. PIV data collection was synchronized with the same induction sensor on the traverse track that triggered the profile deceleration.

Raw images were pre-processed with a min/max contrast normalization filter of size $14 x 14$ pixels and a sliding-average subtraction filter of 50 pixel width. Velocity fields were calculated with a multi-grid/multipass cross-correlation algorithm using DaVis software (LaVision, v8.1.2). Velocity fields were averaged across 9 trials.

\section{Numerical Method}

A set of preliminary two-dimensional simulations generated quantitative predictions of the model perching problem described in section III. The Boundary Data Immersion Method (BDIM), a robust immersed 
boundary method suitable for dynamic fluid-structure interaction problems detailed in Weymouth et al. ${ }^{10}$ and Weymouth and Yue, ${ }^{11}$ was used for this purpose. Briefly, the full Navier-Stokes equations and the prescribed body kinematics shown in figure 1 are convolved with a kernel of support $\epsilon=2 h$, where $h$ is the grid spacing. The integrated equations are valid over the complete domain and allow for general solid-body dynamics to be simulated. Previous work has validated this approach for a variety of dynamic rigid-body problems such as translating airfoils ${ }^{4}$ and deforming-body problems. ${ }^{6}$

The Reynolds number based on the initial steady speed $U_{0}$ is set to $R e=\frac{U_{0} L}{\nu}=2000$ for all cases. A non-inertial computational domain is used with dimensions $8 c \times 8 c$, which translates with the body but does not rotate. All cases use the no-slip and no-penetration boundary conditions on the solid/fluid interface. No-penetration conditions are applied on the top and bottom walls and a convection exit condition is used.

The coupled BDIM equations are discretized using a finite-volume method (third-order convection and second-order diffusion) in space and Heun's explicit second-order method in time. An adaptive time-stepping scheme is used to maintain stability. Table 1 presents a grid convergence study on the pressure force for the $\bar{\tau}^{*}=1 / 4$ test case. The results converge with better than second-order accuracy overall and the maximum difference in the solution between the fine and intermediate grid is only $7 \%$. This verifies the high accuracy of these viscous two-dimensional simulations and the fine grid level is used for the remainder of the paper.

Table 1: Numerical predictions for the peak pressure force lift and drag coefficients for $\bar{\tau}^{*}=1 / 4$ as a function of the grid size $h$ relative to the chord $c$.

\begin{tabular}{cccc}
\hline \hline$h / c$ & 0.04 & 0.02 & 0.01 \\
\hline$C_{p L}$ & 1.26 & 1.68 & 1.81 \\
$C_{p D}$ & 0.85 & 1.13 & 1.22 \\
\hline
\end{tabular}

\section{Results}

In section A, results from direct force measurements in the unsteady and quasi-steady cases are presented. These are compared to the analytical non-circulatory force model (Eq. 15), as well as to forces from lower Reynolds number simulations. In section B, velocity-field visualizations are presented from two test cases $\left(\bar{\tau}^{*}=1 / 4\right.$ and $\left.1 / 2\right)$ using data from PIV at $R e=22000$ and BDIM simulations at $R e=2000$.

\section{A. Force Measurements}

The magnitude of both lift and drag increases with $\bar{\tau}^{*}$ (figure 3). The airfoil experiences a single peak in lift between $t^{*}=0.4$ and $t^{*}=0.5$. This peak increases in magnitude with $\bar{\tau}^{*}$. Lower values of $\bar{\tau}^{*}$ approach quasi-steady predictions.

During the rotational phase, the airfoil experiences a large spike in drag around $t^{*}=0.5$, as shown in figure 3. The peak decays and drag transitions to a thrust around $t^{*}=0.6$. The relative magnitude of the peak thrust relative to the peak drag increases with $\bar{\tau}^{*}$, with $\bar{\tau}^{*}=1 / 32$ exhibiting no thrust while $\bar{\tau}^{*}=1 / 2$ exhibits a peak thrust $3 / 4$ the magnitude of the peak drag. At small $\bar{\tau}^{*}$, the results approach quasi-steady predictions.

Non-circulatory (added-mass) forces (Eq. 15) are overlaid on the measured forces (figure 3). Measured peak lift and drag are much greater than non-circulatory forces. Deviations are most significant at $t^{*}=0.5$, where non-circulatory lift and drag are near 0 while measured lift and drag are close to maximal. Results are closest to the non-circulatory model near the start and end of the maneuver.

Numerical results from the $R e=2000$ simulations are qualitatively similar, but show some quantitative differences (figure 4). The simulations are in phase with experiment, displaying peak lift and drag at around $t^{*}=0.4$ and $t^{*}=0.5$, respectively. Numerical results also show a peak thrust at around $t^{*}=0.8$, which is close in phase to the experimental results for $\bar{\tau}^{*} \leq 1 / 4$ but slightly off for $\bar{\tau}^{*}=1 / 2$. 

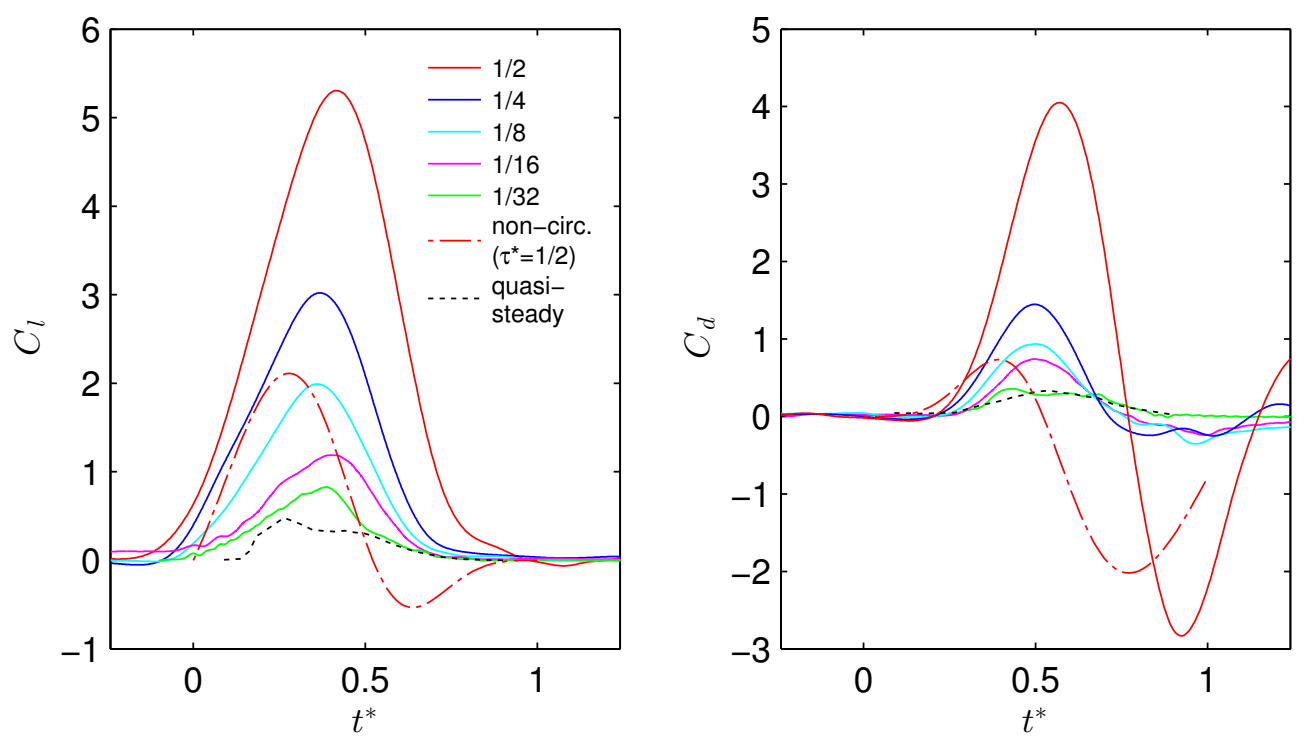

Figure 3: Lift and drag coefficients in non-dimensionalized time, plotted for varying $\bar{\tau}^{*}$. The magnitudes of forces increase with $\bar{\tau}^{*}$. At lower $\bar{\tau}^{*}$, results approach quasi-steady predictions (dotted black line). The predicted non-circulatory (added-mass) force for $\bar{\tau}^{*}=1 / 2$ (Eq. 15) is overlaid as a dotted red line. The measured forces (solid red line) are well above these predictions. Lift coefficients are more than twice the noncirculatory predictions for $\bar{\tau}^{*}=1 / 2$, and are positive for the entire maneuver. Peak drag is over four times the non-circulatory force, but the parasitic thrust is greater than non-circulatory predictions. Nevertheless, this observed parasitic thrust is counteracted by a much larger drag earlier in the maneuver.
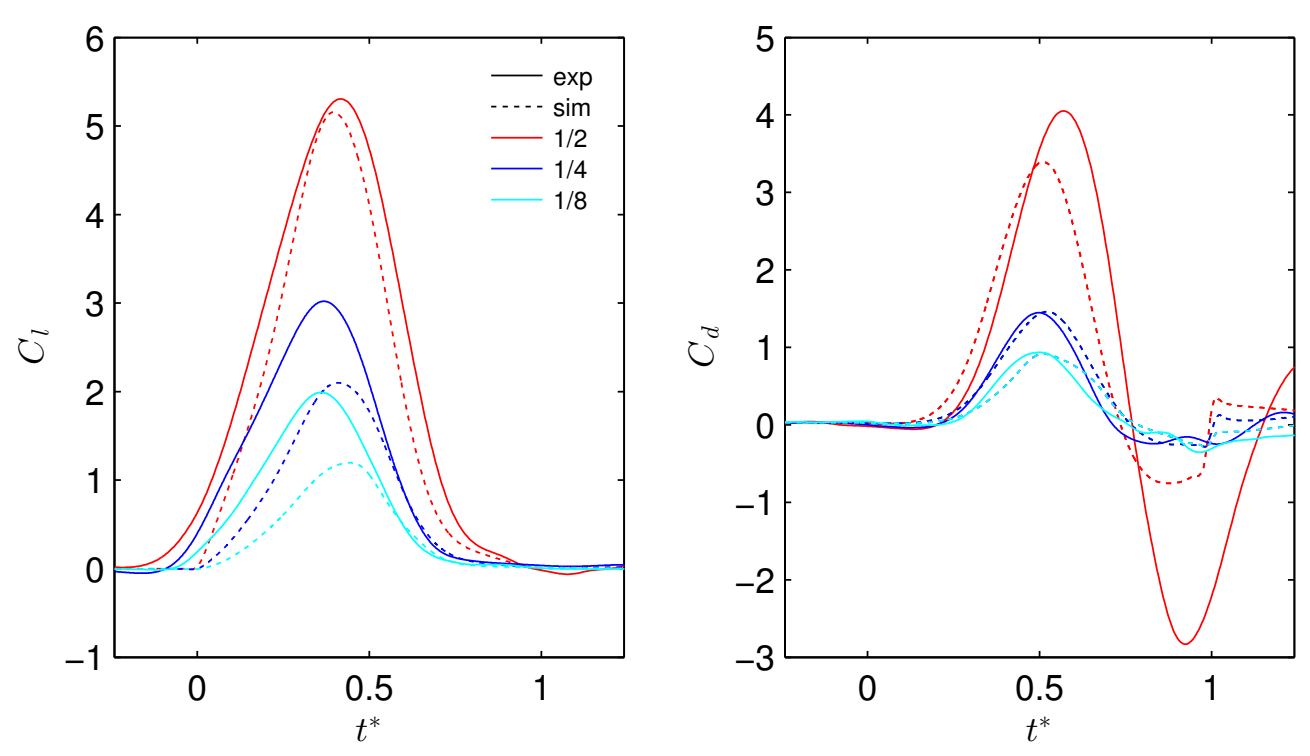

Figure 4: A comparison between measured force results (solid lines) at $R e=22000$ and simulated results (dotted lines) at $R e=2000$ for $1 / 8 \leq \bar{\tau}^{*} \leq 1 / 2$. The phasing and magnitudes of drag are similar between simulations and experiment. The phasing for lift is similar as well, however magnitudes of lift are similar only in the $\bar{\tau}^{*}=1 / 2$ case, due to Reynolds-number effects. 
The magnitude of simulated lift is similar to measured lift for $\bar{\tau}^{*}=1 / 2$. However, peak lift for $\bar{\tau}^{*}=1 / 4$ is about $2 / 3$ of the measured value, while for the $\bar{\tau}^{*}=1 / 8$ case it is about $1 / 2$ of the measured value, due to Reynolds-number effects. The magnitude of peak drag is very similar between simulations and measurements. Peak thrust is similar between simulations and experiment for $\bar{\tau}^{*}=1 / 4$ and $1 / 8$, but is reduced compared to experiment for $\bar{\tau}^{*}=1 / 2$. The rapid jump in the simulated drag time-history at $t^{*}=1$ is due to simulating the discontinuous deceleration profile (figure 1).

\section{B. Velocity-Field Visualizations}

Figure 5 shows the vorticity, derived from PIV at $R e=22000$ and simulations at $R e=2000$. For each $R e$, results from $\bar{\tau}^{*}=1 / 4$ and $1 / 2$ are shown. For the higher $R e$ case at either $\bar{\tau}^{*}$, a strong leading-edge vortex (LEV) forms. This LEV grows in size until separation occurs. Qualitatively, there is no apparent distinction in the size and strength of the LEV between the two $\bar{\tau}^{*}$. During the motion, a streak of negative sign (counterclockwise) vorticity sheds continuously off the trailing edge. A starting vortex (SV) forms at the trailing edge by $t^{*}=0.33$. Towards the end of the maneuver, this vortex rolls up with the negative vorticity shed off the trailing edge, forming a single, larger vortex. This vortex convects in the $-\hat{\mathbf{y}}$ direction. This $\hat{\mathbf{y}}$ displacement from $t^{*}=0.33$ to $t^{*}=1$ is approximately equal in all cases. By $t^{*}=1$, a clockwise stopping vortex has formed at the trailing edge.

Some differences can be seen between $\bar{\tau}^{*}=1 / 4$ and $1 / 2$. The streak of negative vorticity off the trailingedge is shorter but stronger in the $\bar{\tau}^{*}=1 / 2$ case. The trailing-edge wake remains closer to the body at higher $\bar{\tau}^{*}$. Instabilities in the streak are more obvious at the lower $\bar{\tau}^{*}$.

Simulations of the $R e=2000$ case are similar to the $R e=22000$ PIV measurements in several ways (figure 5). For both shape change numbers, an LEV and SV form. A streak of negative vorticity is shed off the trailing edge throughout the maneuver. At the end of the maneuver, a stopping vortex forms.

As in the higher-Re cases, there are more pronounced instabilities in the trailing-edge vorticity streak for the lower shape change number, and the starting vortex and streak are stronger and more coherent at the larger shape change number. The SV also convects in the $-\hat{\mathbf{y}}$ direction by a constant distance.

In contrast to the high Re cases, there are pronounced differences in the size of the LEV between $\bar{\tau}^{*}=1 / 4$ and $1 / 2$. In particular, the LEV forms more quickly at the lower shape change number. LEV separation begins around $t^{*}=0.5$ for $\bar{\tau}^{*}=1 / 4$ but is delayed until about $t^{*}=0.67$ for $\bar{\tau}^{*}=1 / 2$. Separation of positive vorticity near the trailing edge is observed at $t^{*}=0.67$, but only at the lower $\bar{\tau}^{*}$.

\section{Discussion}

The measured and simulated forces are well above non-circulatory potential flow predictions (figures 3 and 4). Furthermore, while non-circulatory forces can only provide positive lift for half the maneuver, the experimentally-derived lift is positive for the majority or all of the maneuver, especially at large $\bar{\tau}^{*}$. The non-circulatory drag was also predicted to be overwhelmed by parasitic thrust, but the measurements reveal that, while thrust is present, it is not dominant. Circulatory effects, which were omitted from analytical calculations, are thought to be responsible for these discrepancies.

Figure 5 shows that the trailing-edge wake is composed entirely of negative vorticity. Positive vorticity is largely constrained to the LEV or else is distributed near the body. Due to Kelvin's theorem, the net circulation of the system must always be zero. Thus the total positive circulation close to the body is equal and opposite to the negative circulation in the trailing-edge streak. If we treat the wake and LEV as a superposition of vortex pairs, with the positive vortex in the LEV or near the body and the negative vortex in the wake, we can gain insight into the circulatory generation of forces (figure 6). This is similar to the method used by von Kármán and Sears in describing wake dynamics for a flat-plate airfoil. ${ }^{12}$

Any vortex pair with net zero circulation in a two-dimensional flow has an associated hydrodynamic impulse, ${ }^{13}$

$$
\mathbf{I}=\Gamma r \hat{\mathbf{i}},
$$

where $\Gamma$ is the circulation of one of the vortices, $r$ is the distance between vortex centres, and $\hat{\mathbf{i}}$ is a unit vector perpendicular to a line connecting the vortex centres, and points along the average direction of fluid motion between the vortex pair. Forces acting on the body come about in part from a reaction to the rate of change of hydrodynamic impulse, ${ }^{13}$ as $\mathbf{F}=-\rho \dot{\mathbf{I}}$. From Eq. (17), this results in the relation

$$
\mathbf{F}=-\rho \dot{\Gamma} r \hat{\mathbf{i}}-\rho \Gamma \dot{r} \hat{\mathbf{i}}
$$



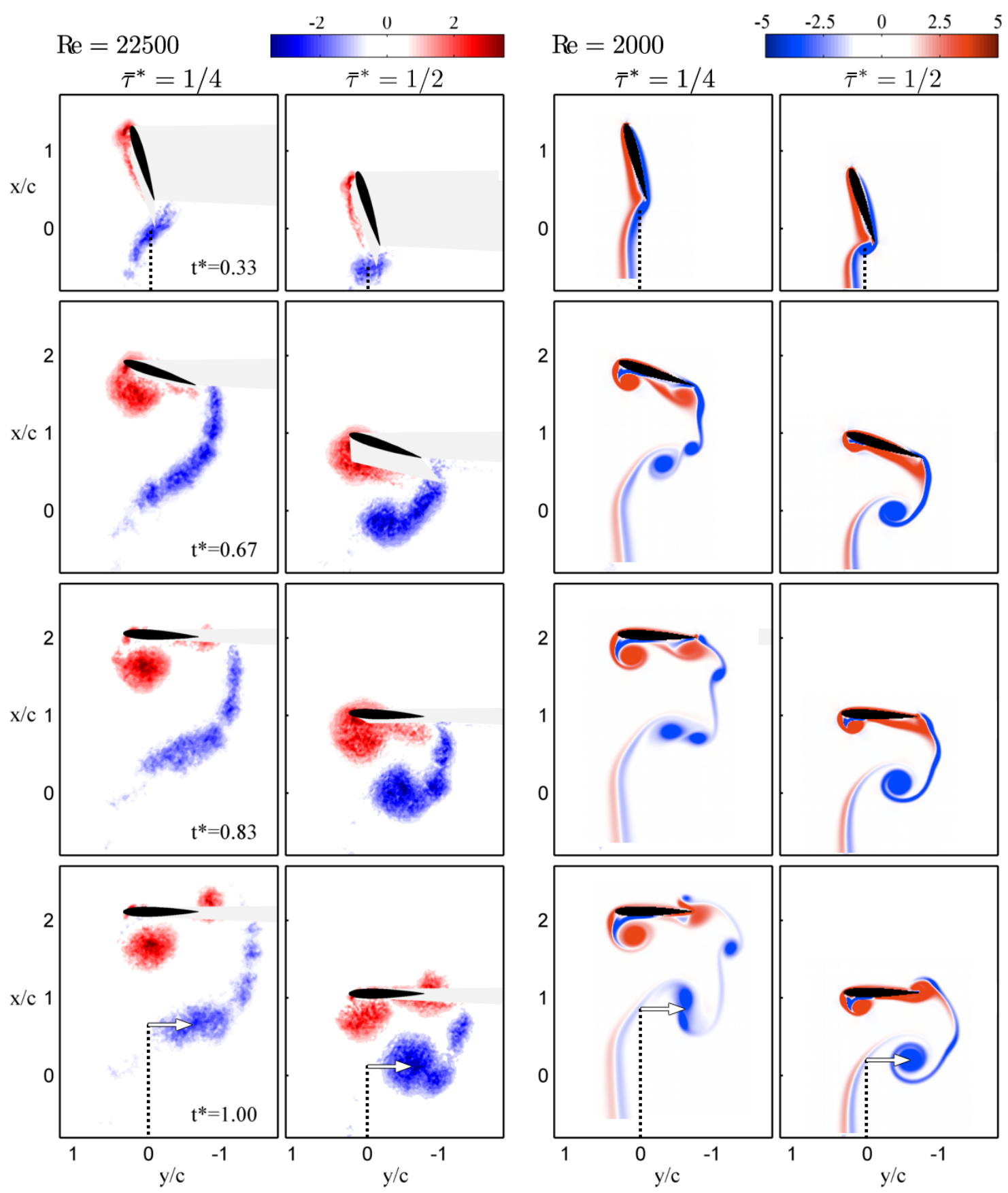

Figure 5: PIV (left) and simulated (right) vorticity fields show that the wing section generates vorticity at the trailing and leading edge. This generation of vorticity, and the relative convection of coherent structures, generates the large forces observed in figures 3 and 4 . The airfoil is translating in the positive $\mathrm{x}$-direction. Columns 1 and 2 (from the left) are PIV at $R e=22000$, while columns 3 and 4 are flow field simulations $R e=2000 . \bar{\tau}^{*}=1 / 4$ and $1 / 2$ are shown for each $R e$. Vorticity is non-dimensionalized by $\omega^{*}=\omega c / U_{0}$. The dotted line at $t^{*}=0.33$ and 1 shows the approximate starting $y$ position of the starting vortex, and the arrows at $t^{*}=1$ indicate their displacement in $y$. This displacement is similar across $\bar{\tau}^{*}$ and Re. By Eq. (20), this indicates that this circulatory drag should scale with $\bar{\tau}^{*}$. In the low-Re cases, separation of positive vorticity near the trailing-edge can be seen at $t^{*}=0.67$ and $\bar{\tau}^{*}=1 / 4$, but not for $\bar{\tau}^{*}=1 / 2$. The reduced separation at higher $\bar{\tau}^{*}$ would increase lift. 
Eq. (18) shows that vortex forces can be altered through the addition or elimination of circulation to or from the vortex pair $(\dot{\Gamma})$ and by the relative convection of a vortex in a pair relative to the other $(\dot{r})$.

The generation of circulation $(\dot{\Gamma})$ occurs only at the surface of the body. During most of the maneuver, generation of negative vorticity is constrained to the trailing edge, while generation of positive vorticity is mostly constrained to the leading edge (figure 5). Thus, at the instant of vortex generation, $\hat{\mathbf{i}}$ is oriented normal to the airfoil's chord (figure 6). Early in the maneuver, the net force points towards the suction side, generating a net lift and drag. As the foil slows, this production rate drops to zero.

The total vorticity in the wake by $t^{*}=1$ appears approximately equal at $\bar{\tau}^{*}=1 / 4$ and $1 / 2$ at a given $R e$. This vorticity, along with equal positive vorticity near the leading edge, is generated in time $T$, the period of rotation and deceleration. A total force will be produced, as

$$
F=\rho \dot{\Gamma} c \sim \rho \frac{\Gamma_{\max }}{T} c=\rho \Gamma_{\max } U_{0} \bar{\tau}^{*},
$$

showing how both lift and drag could depend on $\bar{\tau}^{*}$ through to the generation of circulation.

The remaining contributions to force in Eq. (18) come about from $\dot{r}$. This is manifested in figure 5 and sketched in figure 6 by the displacement of the trailing-edge wake relative to positive circulation near the body. We see from figure 5 that the trailing-edge streak translates predominantly in the $-\hat{\mathbf{y}}$ direction, generating drag. This would counteract the low drag observed in the non-circulatory model (figure 4).

For $\bar{\tau}^{*}=1 / 4$ and $1 / 2$ at both $R e=22000$ and 2000, the distance of starting vortex convection in the $y$ direction is approximately equal (figure 5). Denoting the convection distance by $d_{y}$ as in figure 6 , and noting that it occurs in a constant time period $\sim T$, we find that the induced $y$ velocity of the starting vortex $\left(v_{\text {ind }}\right)$ is

$$
v_{\text {ind }} \sim \frac{d_{y}}{T}=\bar{\tau}^{*} U_{0} \frac{d_{y}}{c} .
$$

This would yield a drag $F_{d} \sim \rho \Gamma U_{0} \frac{d_{y}}{c} \bar{\tau}^{*}$, indicating that this circulatory contribution to drag should scale with the shape change number.

In the simulated flowfield (figure 5), we observe increased separation of positive vorticity near the body at the lower shape change number. The low pressure suction from this positive vorticity would primarily increase lift. Weymouth et al. ${ }^{14}$ introduced a deflation scaling parameter, which for an object undergoing rapid area change is proportional to

$$
\sigma^{*} \propto \tau^{*} \sqrt{R e}
$$

A higher value of this parameter indicates increased separation delay. We expect that lift would be enhanced if the separation of the positive boundary layer vorticity is delayed until the end of the maneuver. This parameter predicts that the delay depends strongly on $\tau^{*}$, providing another means by which lift could scale with the shape change number.

Eq. (21) also predicts that separation delay depends on $R e$, but only to the power of $1 / 2$ while the dependence on $\bar{\tau}^{*}$ is to the first power. This helps reconcile the discrepancy between simulated and measured lift (figure 4), as the low Re simulations display reduced lift compared to the high Re measurements, but the relative discrepancy decreases with increasing $\bar{\tau}^{*}$. Still, differences in lift between the higher and lower $R e$ cases may also be due to effects of transition at the higher Re, versus laminar flow at the lower $R e$.

An expanding and decelerating body experiences negative effects from non-circulatory forces, in contrast to a deflating and accelerating body. However, the above heuristic arguments show how rapid area change can generate positive lift and drag at all times though the manipulation of circulatory forces, counteracting the parasitic added-mass thrust and negative lift seen in figure 3. Rigorous validation of these ideas awaits their implementation into a robust and more comprehensive analytical model, which could be tested on the current data. 

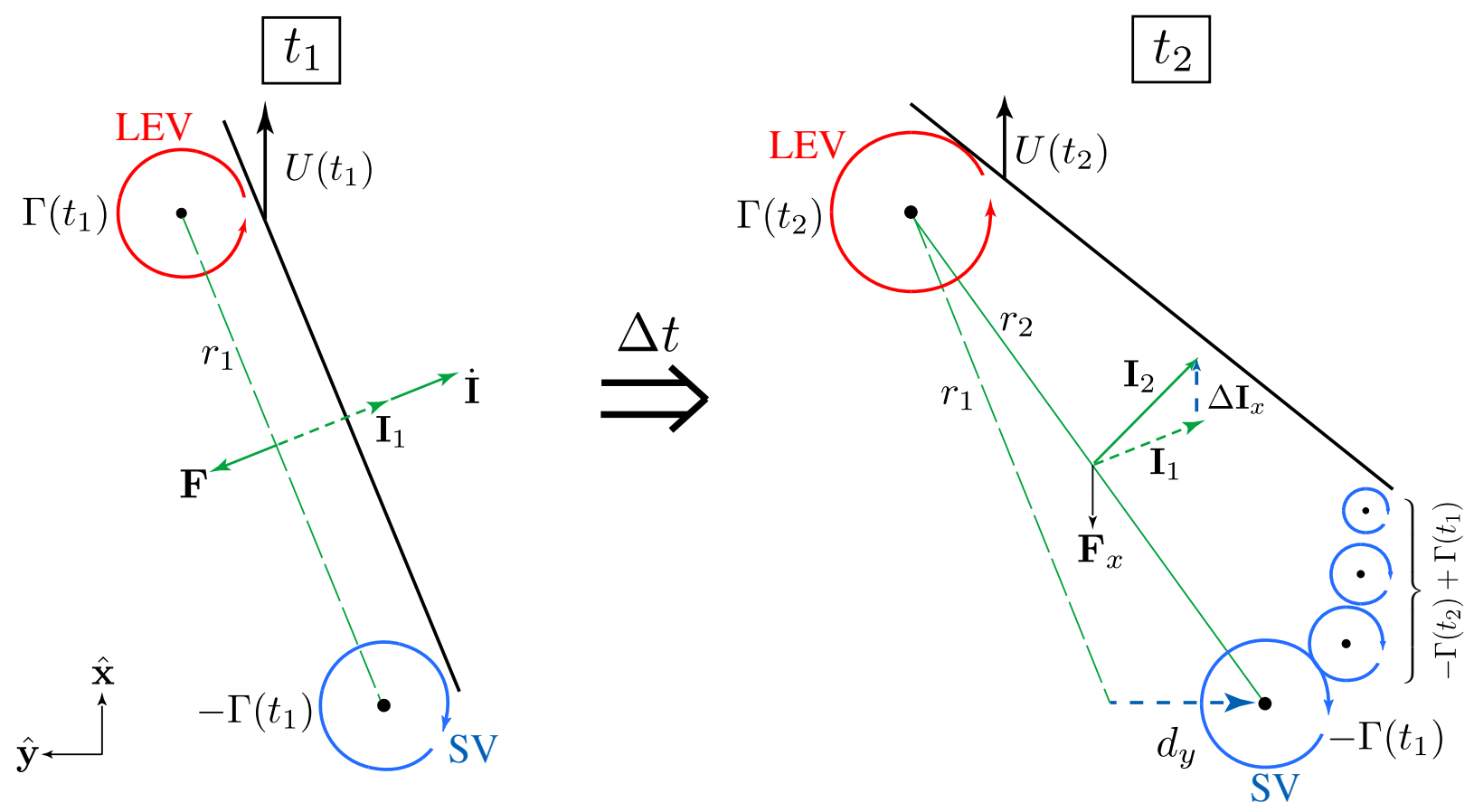

Figure 6: Sketch of how the manipulation of vortex impulse generates forces. The orientation of the airfoil is the same as in figure 5. At $t_{1}$, vortices are formed at the trailing and leading edge. The total impulse $\left(\mathbf{I}_{1}\right)$ of the vortex pair is the distance between these vortices $\left(r_{1}\right)$ multiplied by the positive circulation of one vortex $\left(\Gamma\left(t_{1}\right)\right)$. The rate of change of the impulse $(\dot{\mathbf{I}})$ through the generation of circulation causes a reaction force $\mathbf{F}$ on the body. At $t_{2}$, the airfoil has rotated and translated forward. The leading-edge vortex circulation has increased, while the circulation of the starting vortex has not changed. However, the two vortices have convected apart by a distance $d_{y}$ in the $y$ direction. This has increased the impulse by $\Delta \mathbf{I}_{y}$. As a result, the airfoil has experienced a reaction drag force $\mathbf{F}_{x}$. Additional forces are produced from the generation and convection of the remaining circulation in the wake.

\section{Conclusions}

Flying animals execute high-precision maneuvers such as perching through rapid changes in wing area. Yet, from an aerodynamic perspective, it is unclear how they can produce high lift and drag simultaneously. As a simple perching model, we measured the forces and flowfields associated with simultaneously decelerating and rotating an airfoil section through direct force measurements, Particle Image Velocimetry (PIV) and simulations using the Boundary Data Immersion Method. Analytical arguments were used to show that added-mass forces should scale with the shape change number, $\bar{\tau}^{*}$, a ratio between velocity of expansion and initial velocity. The added-mass forces were predicted to yield significant peaks in lift and drag, but were also predicted to generate forces counterproductive to perching, including a negative lift and parasitic thrust. However, measured forces were well above non-circulatory predictions. Additionally, the lift was positive throughout the maneuver, and though added-mass thrust was present, significantly greater drag was observed earlier in the maneuver. These forces were observed to scale with shape change number, showing that rapid area change can increase force generation in the case of a rapidly rotating and decelerating airfoil.

PIV and the simulated velocity field revealed large vortical structures near the body and in the wake. Circulatory effects could generate forces through the generation of vorticity and the manipulation of the relative position and distribution of negative vorticity in the wake. These forces, in view of the total circulation and the relative movement of vortices in the flow field, were predicted to generate positive lift and drag throughout the maneuver. Additionally, they were predicted to scale with $\bar{\tau}^{*}$. Thus, wake manipulation through rapid area change can circumvent counterproductive non-circulatory effects in airfoil pitching and deceleration. Rapid area change may therefore be a means by which a perching bird can maintain lift while quickly braking to a controlled stop. 


\section{Acknowledgments}

The authors wish to thank the Natural Sciences and Engineering Research Council of Canada for financial support.

\section{References}

${ }^{1}$ Cory, R. and Tedrake, R., "Experiments in fixed-wing UAV perching," Proceedings of the AIAA Guidance, Navigation, and Control Conference, Honolulu, Hawaii, August 2008.

${ }^{2}$ Berg, A. and Biewener, A., "Wing and body kinematics of takeoff and landing flight in the pigeon (Columbia livia)," Journal of Experimental Biology, Vol. 213, 2010, pp. 1651-1658.

${ }^{3}$ Carruthers, A., Thomas, A., Walker, S., and Taylor, G., "Mechanics and aerodynamics of perching manoeuvres in a large bird of prey," Aeronautical Journal, Vol. 114, No. 1161, 2010, pp. 673-680.

${ }^{4}$ Wibawa, M. S., Steele, S. C., Dahl, J. M., Rival, D. E., Weymouth, G. D., and Triantafyllou, M. S., "Global vorticity shedding for a vanishing wing," Journal of Fluid Mechanics, Vol. 695, 2012, pp. 112-134.

${ }^{5}$ Weymouth, G. and Triantafyllou, M. S., "Global vorticity shedding for a shrinking cylinder," Journal of Fluid Mechanics, Vol. 702, 2012, pp. 470-487.

${ }^{6}$ Weymouth, G. and Triantafyllou, M. S., "Ultra-fast escape of a deformable jet-propelled body," Journal of Fluid Mechanics, Vol. 721, 2013, pp. 367-385.

${ }^{7}$ Katz and Plotkin, Low-Speed Aerodynamics, Cambridge University Press, 2001.

${ }^{8}$ Theodorsen, T., "General theory of aerodynamic instability and the mechanism of flutter," NACA Report $496,1935$.

${ }^{9} \mathrm{Ol}$, M. V., Bernal, L., Kang, C.-K., and Shyy, W., "Shallow and deep dynamic stall for flapping low Reynolds number airfoils," Experiments in Fluids, Vol. 46, 2009, pp. 883-901.

${ }^{10}$ Weymouth, G., Dommermuth, D., Hendrickson, K., and Yue, D.-P., "Advancements in cartesian-grid methods for computational ship hydrodynamics," Proceedings of the 26th Symposium on Naval Hydrodynamics, Rome, Italy, 17-22 September 2006.

${ }^{11}$ Weymouth, G. and Yue, D.-P., "Boundary data immersion method for cartesian-grid simulations of fluid-body interaction problems," Journal of Computational Physics, Vol. 230, No. 16, 2011, pp. 6233 - 6247.

12 von Kármán, T. and Sears, W. R., "Airfoil theory for non-uniform motion," Journal of Aeronautical Sciences, Vol. 5, 1938, pp. 379-390.

${ }^{13} \mathrm{Wu}$, J., Ma, H., and Zhou, J., Vorticity and Vortex Dynamics, Lecture notes in mathematics, Springer Berlin Heidelberg, 2006.

${ }^{14}$ Weymouth, G. D., Subramaniam, V., and Triantafyllou, M. S., "Octopus-like robot evades drag and recovers energy to power an ultra-fast escape," Physical Review Letters, under review. 